\title{
Electron energy spectrum in a spherical quantum dot with smooth confinement
}

\author{
V. Holovatsky, O. Voitsekhivska, V. Gutsul \\ Chernivtsi National University \\ 2, Kotsyubynsky str., 58012 Chernivtsi, Ukraine; e-mail: theorphys@chnu.cv.ua
}

\begin{abstract}
The electron energy spectrum in a quantum dot (QD) with smooth dependences of the quasiparticle potential energy and the effective mass at the interface between semiconductor media is calculated in the effective mass approximation. It is shown that the electron energy corrections due to the tailing of the interface are nonmonotonous functions of the QD radius, the increasing of which brings to the rapid increasing of shifts, reaching their maxima, and slowly decreasing for the QDs of big sizes. The calculations prove that the relative corrections for the different electron energy levels in a spherical QD are placed closer to each other with increase in the radius. The growth of the parameter of interface tailing leads to the proportional increase in the corrections to electron energy spectra. Numerical calculations are performed for $\mathrm{HgS} / \mathrm{CdS}$ and $\mathrm{GaAs} / \mathrm{Al}_{\mathrm{x}} \mathrm{Ga}_{1-\mathrm{x}} \mathrm{As}$ QDs, all dependences being qualitatively similar.
\end{abstract}

Keywords: quantum dot, smooth confinement, interface, electron energy spectrum.

Manuscript received 07.02.07; accepted for publication 27.09.07; published online 31.10.07.

\section{Introduction}

The wide perspectives of the utilization of semiconductor nanoheterosystems in laser and nanoscheme computer technique determine the intensive development of the physics of quantum wells, wires, and dots, though the physical phenomena in such objects are often essentially different from those having place in bulk crystals. Therefore, the investigation of nanoheterosystems is of great interest for the scientists.

The great number of theoretical and experimental researches has been performed in this branch of physics till now. The modern technological methods allow one to produce the high-quality nanoheterostructures of various shapes created of different semiconductor materials. The experimental investigations stimulate the intensive theoretical study of physical phenomena in such nanosystems.

Problems of the establishment of a theory describing the physical phenomena in nanoheterosystems are related to the fact that these objects are so small that the size quantization becomes essential. Nevertheless, the microscopic approach taking into account the atomic-molecular structure of a heterosystem is very complicated for such systems; it is used only for the nanocrystals containing several hundreds of atoms. At the same time, the effective mass approximation and the dielectric continuum model introduced for the description of physical processes running in bulk semiconductor crystals are often used in nanophysics after some modifications.

The skiplike rectangular potential or the parabolic one of a harmonic oscillator (see Fig. 1) (for which there are the exact solutions of the Schrödinger equation) is usually used in the calculations of quasiparticle energy spectra in spherical quantum dots (QDs). Nevertheless, the simplicity of the solutions has some disadvantages in the description of potentials.

The main disadvantage of parabolic potential is the neglect of the difference between the quasiparticle effective mass inside a QD and in the external medium. Moreover, it is impossible to obtain the continuum energy spectrum of quasiparticles with high energies due to the fact that this potential is not confined at infinity. The authors of [1], taking into account the abovementioned disadvantages of the classical parabolic potential, used a model potential. It is equal to the parabolic one inside the QD, and it is constant in the external medium. The calculation of the energy spectrum is rather difficult and performed within some approximations. 


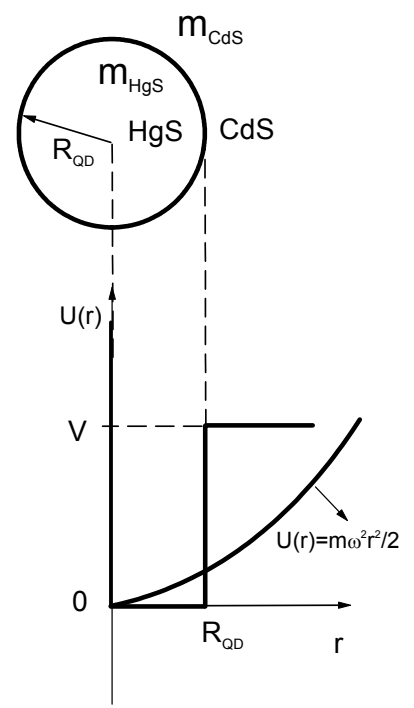

Fig. 1. Geometrical scheme of spherical quantum dots and schemes of the skiplike rectangular potential and the potential of a harmonic oscillator.

The other disadvantage of the rectangular potential model is the unphysical skiplike behavior of the potential energy and the effective mass at the interface of semiconductor media. The same unphysical features are observed in the research of electrostatic image forces due to the skiplike varying of the dielectric constant at the media interface. In works [2, $3]$, the potential of image forces and corrections to the electron binding energies were calculated by using the smooth functions for the dielectric constants such as

$\varepsilon(z)=\frac{\varepsilon_{1}+\varepsilon_{2}}{2}\left[1+\frac{\varepsilon_{2}-\varepsilon_{1}}{\varepsilon_{1}+\varepsilon_{2}} \tanh \left(\frac{z}{L}\right)\right]$.

In work [2], it was shown that the chosen form of the diffuse tailing of the interface of media is proved by experiment. In the approach proposed in [3-5] for the interface between a spherical quantum dot and the external medium, the smooth functions

$$
\begin{aligned}
& f(r)=\tanh \left(\frac{r-R_{0}}{L}\right), \\
& f(r)=\frac{2}{\pi} \arctan \left(\frac{r-R_{0}}{L}\right),
\end{aligned}
$$

and the linear approximating function

$$
f(r)=\left\{\begin{array}{cc}
-1, & r<R_{0}-\frac{L}{2} \\
\frac{r-R_{0}}{L}, & R_{0}-\frac{L}{2}<r<R_{0}+\frac{L}{2}, \\
1, & R_{0}+\frac{L}{2}<r
\end{array}\right.
$$

where $L$ is the parameter characterizing the interface tailing width, were used.

In works $[4,5]$, it was shown that the results of calculations of energy spectra are less sensitive to the form of the approximating function than to the width of the transition layer $L$.

In this paper, the corrections to the energies of spherically symmetric stationary states of an electron which arise due to accounting the tailing of the potential energy and the effective mass functions at the interface of the QD are calculated. The functional dependences of the potential energy of an electron and its effective mass on the distance from the center of a spherical QD are assumed to be as follows:

$U(r)=\frac{V}{2}\left[1+\tanh \left(\frac{r-R_{0}}{L}\right)\right]$,

$m(r)=\frac{m_{1}+m_{2}}{2}\left[1+\frac{m_{2}-m_{1}}{m_{1}+m_{2}} \tanh \left(\frac{r-R_{0}}{L}\right)\right]$.

Here, $m_{1}$ and $m_{2}$ are, respectively, the electron effective masses in a QD and in the external medium, and $V$ is some parameter.

Using the smooth functions $m(r)$ and $U(r)$ is more physical because, in addition to the presence of the transition layer at the interface, the macroscopic parameters - effective mass $(m)$ and potential energy $(U)$ - at a fixed point of the nanosystem are the results of the averaging which cannot be skiplike.

The energy spectrum of a quasiparticle with the effective mass (6) in the potential well (5) is found from the solution of the Schrödinger equation

$\left(-\frac{\hbar^{2}}{2} \vec{\nabla} \frac{1}{m(r)} \vec{\nabla}+U(r)\right) \Psi(\vec{r})=E \Psi(\vec{r})$.

It is obvious that this equation cannot be solved exactly; therefore, we have to use some approximating methods. For example, the ground state energy can be calculated in the framework of the variational method, but the problem becomes quite difficult for the energies of excited states.

We propose to solve Eq. (7) by using the approximation of the smooth functions (5) and (6) by the skiplike functions shown in the Fig. 1. These functions give the opportunity to obtain the exact solution of the respective Schrödinger equation. When the number of skips is big enough, the obtained solutions will be sufficiently close to the solutions of Eq. (7) with the smooth functions (5) and (6). Thus, the smooth interface is substituted by several imaginary intermediate layers with effective masses $m_{i}$ and potentials $V_{i}$. Then the calculations are carried out on the base of the conditions of equality of the areas under the smooth functions (5) and (6) and the corresponding skiplike functions which are shown in Fig. 2. The problem of solving the Schrödinger equation (7) is the same as the problem to 
find electron energy spectra in complicated spherical semiconductor nanoheterosystems $[6,7]$.

\section{Solution of the Schrödinger equation}

Taking into account the spherical symmetry of the problem and the skiplike functions for the effective mass $m(r)$ and the potential $U(r)$ chosen as

$U(r)=\sum_{i=0}^{N} V_{i} \sigma_{i}(r), \quad m(r)=\sum_{i=1}^{N} m_{i} \sigma_{i}(r)$

with $\sigma_{i}(r)=\left\{\begin{array}{l}1, \quad r_{i-1} \leq r \leq r_{i} \\ 0, \text { other region }\end{array}, \quad r_{-1}=0\right.$,

it is convenient to write the radial part of the wave function as

$R(r)=\sum_{i=0}^{N} R_{i} \sigma_{i}(r)$.

From the Schrödinger equation (7), we obtain the system of $N+1$ equations for the radial wave functions $R_{i}(r)$

$$
\begin{array}{r}
-\frac{\hbar^{2}}{2 m_{i}} \frac{1}{r^{2}} \frac{\partial}{\partial r}\left(r^{2} \frac{\partial R_{i}(r)}{\partial r}\right)+\left(V_{i}-E\right) R_{i}(r)=0, \\
i=0,1, \ldots N .
\end{array}
$$

Every of them contains the constant potential energy $V_{i}$ and the constant effective mass $m_{i}$. The solutions of Eqs. (10) are the linear combinations of Bessel spherical functions of the first and second orders

$$
R_{i}(r)=A_{i} j_{0}\left(k_{i} r\right)+B_{i} n_{0}\left(k_{i} r\right), \quad i=0,1, \ldots, N,
$$

or the respective Hankel functions of the first and second orders

$R_{i}(r)=A_{i}\left(h_{0}^{-}\left(k_{i} r\right)+S_{i} h_{0}^{+}\left(k_{i} r\right), \quad i=0,1, \ldots, N\right.$,

where $k_{i}=\hbar^{-1} \sqrt{2 m_{i}\left(E-V_{i}\right)}$. For $E<V_{i}$, the Bessel functions of the first and second orders are transformed into the correspondent modified spherical Bessel functions $I_{0}\left(\left|k_{i}\right| r\right)$ and $K_{0}\left(\left|k_{i}\right| r\right)$.

The conditions of continuity of the wave function and its probability current density at all points $r_{i}$ bring to the system of $2(N+1)$ equations

$$
\left.\begin{array}{c}
\left.R_{i}(r)\right|_{r=r_{i}}=\left.R_{i+1}(r)\right|_{r=r_{i}} \\
\left.\frac{1}{m_{i}} \frac{d R_{i}(r)}{d r}\right|_{r=r_{i}}=\left.\frac{1}{m_{i+1}} \frac{d R_{i+1}(r)}{d r}\right|_{r=r_{i}}
\end{array}\right\} \quad i=0,1, \ldots, N
$$

Inserting representation (11) into the system of equations (13), we obtain the system of linear equations for the determination of the coefficients $A_{i}, B_{i}$,

$$
\left.\begin{array}{l}
j_{0}\left(k_{i} r_{i}\right) A_{i}+n_{\ell}\left(k_{i} r_{i}\right) B_{i}-j_{0}\left(k_{i+1} r_{i}\right) A_{i+1}^{0}- \\
-n_{0}\left(k_{i+1} r_{i}\right) B_{i+1}=0 \\
\frac{1}{m_{i}}\left[j_{0}^{\prime}\left(k_{i} r_{i}\right) A_{i}+n_{0}^{\prime}\left(k_{i} r_{i}\right) B_{i}\right]- \\
-\frac{1}{m_{i+1}}\left[j_{0}^{\prime}\left(k_{i+1} r_{i}\right) A_{i+1}+n_{0}^{\prime}\left(k_{i+1} r_{i}\right) B_{i+1}\right]=0
\end{array}\right\},
$$

where

$$
\begin{aligned}
& j_{0}^{\prime}\left(k_{i} r_{i}\right)=\left.\frac{d j_{0}\left(k_{i} r\right)}{d r}\right|_{r=r_{i}}=-k_{i} j_{1}\left(k_{i} r_{i}\right), \\
& n_{0}^{\prime}\left(k_{i} r_{i}\right)=\left.\frac{d n_{0}\left(k_{i} r\right)}{d r}\right|_{r=r_{i}}=-k_{i} n_{1}\left(k_{i} r_{i}\right) .
\end{aligned}
$$

Using the condition that the wave function should be finite at $r \rightarrow 0$ and $r \rightarrow \infty$, two unknown coefficients are fixed as:

$$
\begin{aligned}
& B_{0}=0, \\
& B_{N+1}=0 .
\end{aligned}
$$

The other unknown $2(N+1)$ coefficients are determined from the system of equations (14) and the normalizing condition

$\int_{0}^{\infty}|R(r)|^{2} r^{2} d r=1$.

Herein, the system of linear equations for the coefficients $A_{i}$ and $B_{i}$ has the nontrivial solutions when the determinant built from Bessel functions of the first and second orders and their derivatives is equal to zero. Thus, we arrive at the dispersion equation for the determination of the electron energy spectrum in a quantum dot with smooth interface.

The same electron energy spectrum can be found using the system of equations (13) with a radial wave function written in the form (12). Using the relation $S_{0}=1$, we obtain the expression for the $S$-matrix $\left(S_{N+1}\right)$, whose poles define the energy of stationary states in the nanosystem $[8,9]$.

\section{Analysis of the results}

The greater the number of skips, the closer are the approximating functions to functions (5) and (6), and the solution of Eq. (7) becomes more exact. In order to define the optimal number of skips $(N)$ in the approximating functions $U(r)$ and $m(r)$, we performed the calculation of the electron energy in spherically symmetric states for various values of $N$.

In Fig. 2, we present the dependences of the electron effective mass and the potential energy in quantum dots $\mathrm{HgS} / \mathrm{CdS}$ and $\mathrm{GaAs} / \mathrm{Al}_{\mathrm{x}} \mathrm{Ga}_{1-\mathrm{x}} \mathrm{As}$ on the distance along the radial coordinate at the fixed number of skips $N=10$ and the tailing parameter $L=1$. 


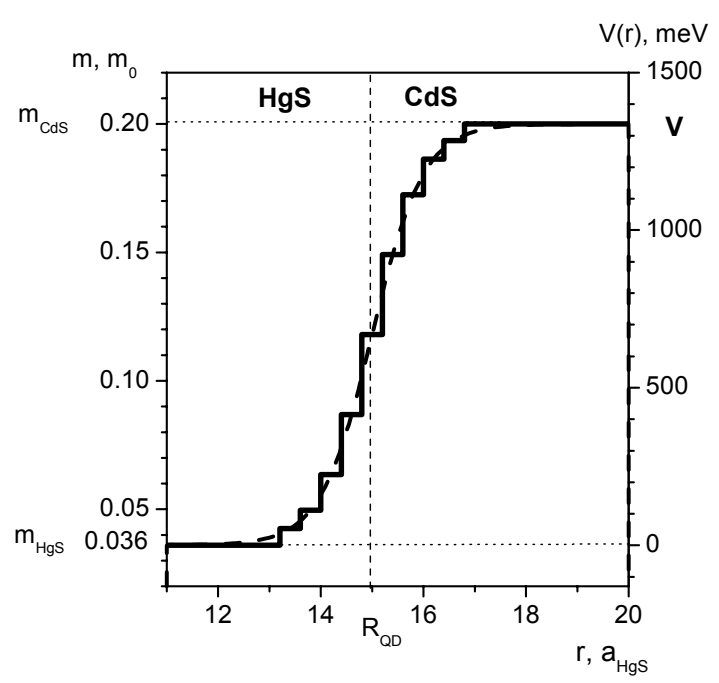

a)

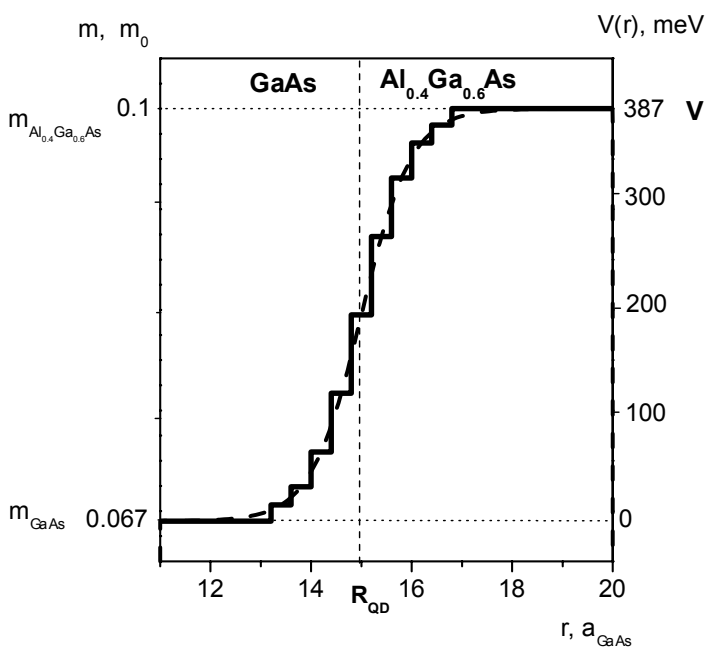

b)

Fig. 2. Dependence of the electron potential energy and the effective mass on the radius $r$ in the $\mathrm{HgS} / \mathrm{CdS}$ (a) and $\mathrm{GaAs} / \mathrm{Al}_{\mathrm{x}} \mathrm{Ga}_{1-\mathrm{x}} \mathrm{As}$ (b) quantum dots. The solid curves are the model skiplike function with $N=10$, and the dashed ones are $m(r)$ and $U(r)$ by formulas (5) and (6).

Parameters of the materials used in the numerical calculations are shown in Table 1.

Table. Parameters of the materials.

\begin{tabular}{|l|c|c|c|}
\hline Material & $\left.m_{e}\left(m_{0}\right)^{*}\right)$ & $a(\stackrel{\mathrm{O}}{)})$ & $V_{e}(\mathrm{eV})$ \\
\hline $\mathrm{HgS}$ & 0.036 & 5.851 & 0 \\
\hline $\mathrm{CdS}$ & 0.2 & 5.818 & 1.35 \\
\hline $\mathrm{GaAs}$ & 0.067 & 5.65 & 0 \\
\hline $\mathrm{Al}_{0.4} \mathrm{Ga}_{0.6} \mathrm{As}$ & 0.1 & & 0.387 \\
\hline
\end{tabular}

*) $\quad m_{0}-$ bare mass of an electron.

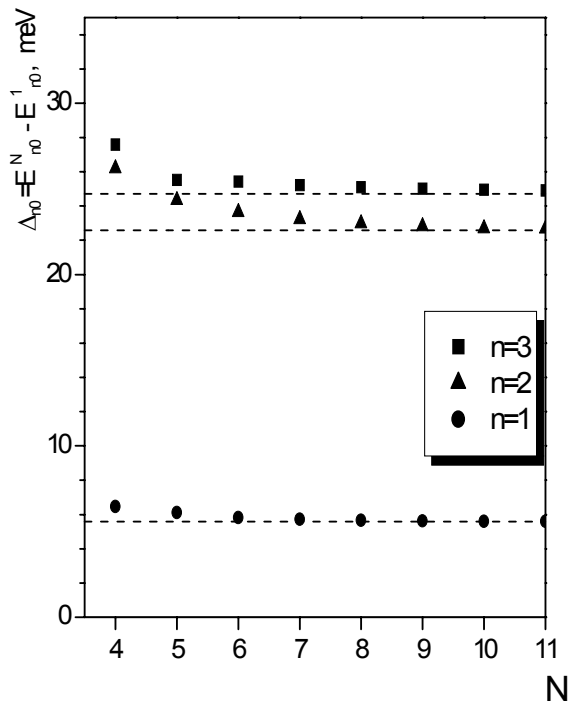

a)

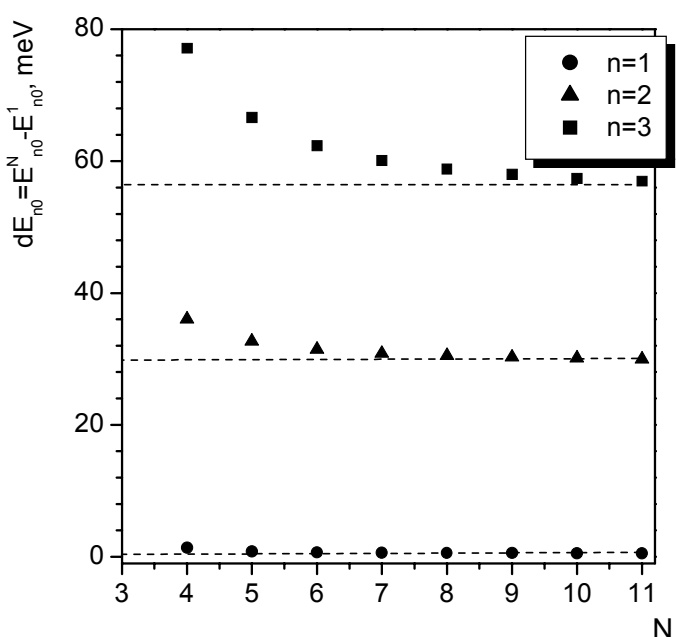

b)

Fig. 3. Dependences of the absolute corrections to the energy levels $E_{10}, E_{20}, E_{30}$ on $N$ in $\mathrm{HgS} / \mathrm{CdS}$ (a) and $\mathrm{GaAs} / \mathrm{Al}_{\mathrm{x}} \mathrm{Ga}_{1-\mathrm{x}} \mathrm{As}$ (b) QDs with the radius $r_{0}=15$.

In Fig. 3, we show the results of calculations of the absolute corrections to the electron energy in spherically symmetric states,

$\Delta_{n 0}=E_{n 0}^{N}-E_{n 0}^{1}$,

where $E_{n 0}^{N}$ - the energies of electron stationary states in a model potential with $N$ skips, and $E_{n 0}^{1}$ - the energies of electron stationary states in a rectangular potential (1 skip).

One can see from Fig. 3 that, as the number $N$ increases, the magnitudes of the absolute corrections are saturated. Herein, for the higher excited states, the saturation is observed at great numbers of skips. The saturation is observed at $N=7-8$ for a $\mathrm{HgS} / \mathrm{CdS}$ QD and at $N=10-11$ for a GaAs/ $/ \mathrm{Al}_{\mathrm{x}} \mathrm{Ga}_{1-\mathrm{x}} \mathrm{As} \mathrm{QD}$. The satisfactory

(C) 2007, V. Lashkaryov Institute of Semiconductor Physics, National Academy of Sciences of Ukraine 
exactness (2-3\%) for all states for both types of quantum dots and for the tailing parameter $L=1 a_{\mathrm{HgS}}$ (a $\mathrm{HgS} / \mathrm{CdS} \mathrm{QD}$ ) and $L=1 a_{\mathrm{GaAs}}\left(\mathrm{a} \mathrm{GaAs} / \mathrm{Al}_{0.4} \mathrm{Ga}_{0.6} \mathrm{As} \mathrm{QD}\right)$ is reached at $N=10$. For the greater value of $L$, the corresponding bigger values of corrections and the electron energy are observed. Therefore, the relative corrections stay the same. Since, the further calculations were performed with the model skiplike functions of the potential and the effective mass at $N=10$.

In Fig. 4, we display the dependence of the electron spectrum on the size of a quantum dot at the different magnitudes of the tailing parameter of the interface. It is seen that the tailing of the interface does not influence the qualitative shape of the dependence of the energy spectrum on the size of a QD.
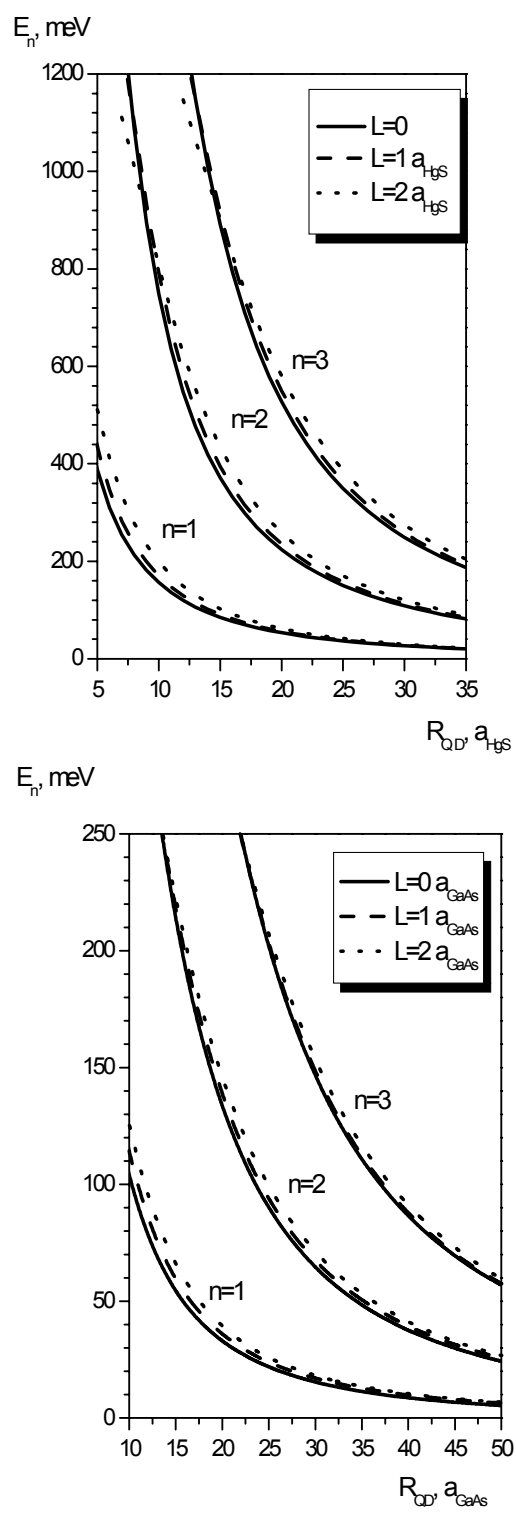

a)

b)

Fig. 4. Dependence of the electron energy in spherically symmetric states $(l=0)$ with $n=1,2,3$ on the radius of $\mathrm{HgS} / \mathrm{CdS}$ (a) and $\mathrm{GaAs} / \mathrm{Al}_{\mathrm{x}} \mathrm{Ga}_{1-\mathrm{x}} \mathrm{As}$ (b) QDs. Solid curves without the interface tailing, dashed curves - at $L=1 a_{\mathrm{HgS}}$, and doted curves - at $L=2 a_{\mathrm{HgS}}$.
In order to evaluate the quantitative influence of the smooth interface between the media on three lowest electron energy levels, we present the results of calculations of the relative corrections to the energies of spherically symmetric states at various values of the parameter $L$ in Fig. 5:

$\delta_{n 0}=\frac{\Delta_{n 0}}{E_{n 0}^{1}}=\frac{E_{n 0}^{N}-E_{n 0}^{1}}{E_{n 0}^{1}}$.

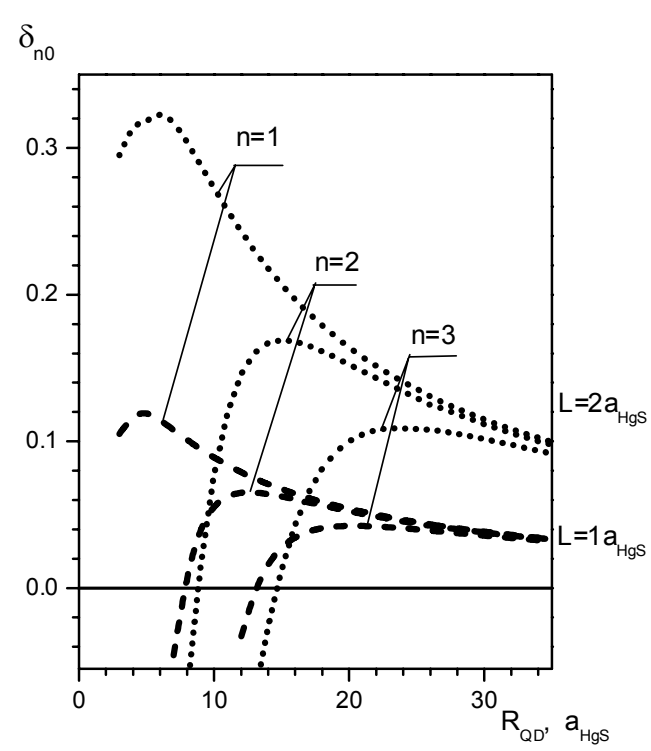

a)

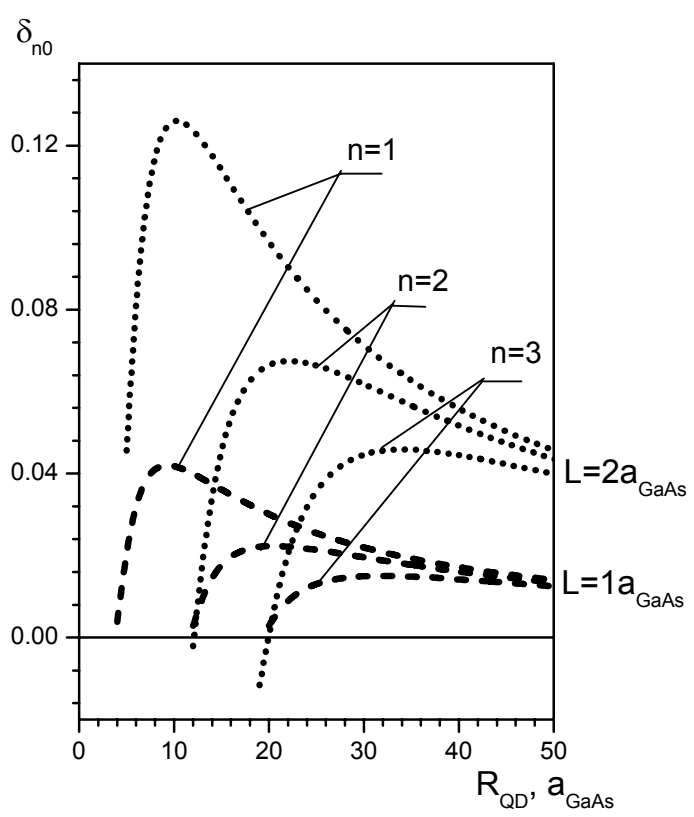

b)

Fig. 5. Dependences of the relative corrections to the energies $E_{n 0}^{1}$ at $n=1,2,3$ on the size of $\mathrm{HgS} / \mathrm{CdS}$ (a) and $\mathrm{GaAs} /$ $\mathrm{Al}_{\mathrm{X}} \mathrm{Ga}_{1-\mathrm{x}} \mathrm{As}$ (b) QDs for different values of the tailing parameter $L$.

\section{(C) 2007, V. Lashkaryov Institute of Semiconductor Physics, National Academy of Sciences of Ukraine}


Figures 4 and 5 prove that the absolute corrections to the ground energy level are the smallest but the relative correction is the biggest. The calculations show that the relative correction to the energies of spherically symmetric states increase with the QD size, and then they decrease and become closer to one another. Such a dependence is understandable because the tailed potential well is thinner near the bottom, and it is wider at the great energies than the respective rectangular well. Therefore, at the small sizes of QDs, the corrections to the energy levels located in the region of energies, where the tailed potential well is wider than that for the rectangular potential, can be negative. The increase in the QD size leads to a decrease of the energies of discrete levels shifted into the region of the potential well where it is thinner than the respective rectangular one, which is accompanied by the increase in the respective energy corrections. The further increase of the QD radius causes a decrease of the influence of the interface tailing, because the probability of the quasiparticle location at the heterointerface decreases rapidly. As a result, the relative corrections for great-size QDs are not sensitive to their radius.

The double increase of the tailing parameter causes the increase of the relative correction by two times. For big QDs, it is somewhat bigger than two.

The account for the smooth behavior of the potential energy and the effective mass at the interface between a QD and the external medium brings to a shift of the energy levels. Its magnitude has the nonmonotonous dependence on the QD size. For rather big QDs, the energy levels are shifted into the region of high energies. Herein, the magnitudes of relative corrections for the different energies of spherically symmetric states are equal. The increase of the tailing parameter causes the proportional increase of relative corrections to the energy levels. All dependences for $\mathrm{HgS} / \mathrm{CdS}$ and $\mathrm{GaAs} / \mathrm{Al}_{\mathrm{x}} \mathrm{Ga}_{1-\mathrm{x}} \mathrm{As}$ quantum dots are qualitatively similar, the quantitative differences are caused by the different values of the effective masses and the different depths of potential wells.

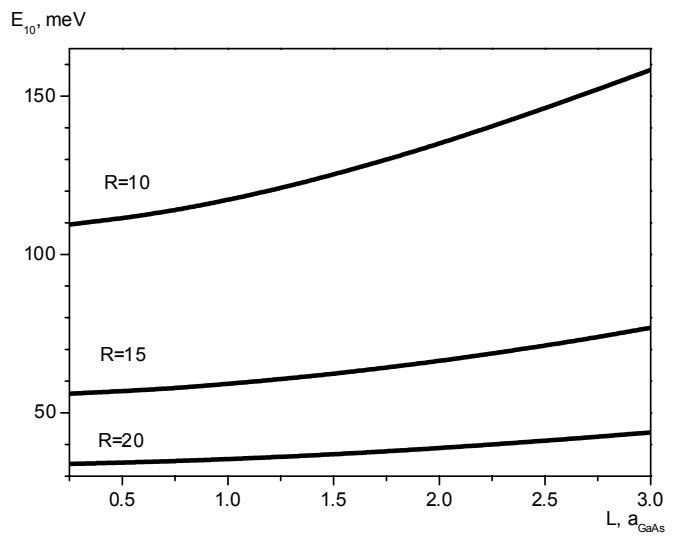

Fig. 6. Dependences of the electron ground state energy on the tailing parameter $L$ in an $\mathrm{Al}_{\mathrm{x}} \mathrm{Ga}_{1-\mathrm{x}} \mathrm{As} \mathrm{QD}$ at a fixed radius $R=(10,15,20) a_{\mathrm{GaAs}}$.
In order to study the influence of the interface tailing on the electron energy spectrum, we calculated the electron ground state energy as a function of the tailing parameter $L$ at various values of the quantum dot radius. The results of calculations are presented in Fig. 6 . As seen, with increase in the tailing parameter, the location of the electron ground energy level is shifted into the region of great energies. Herein, the interface tailing influence is stronger for the quantum dots of small radii. For quantum dots with $R>15 a_{\mathrm{GaAs}}$, the energies of the ground state show almost a linear dependence on the tailing parameter $L$.

\section{Conclusions}

The results of studies allow us to draw the following conclusions.

The skiplike potential at the interface of media used instead of the smooth one gives the opportunity to solve the Schrödinger equation and to calculate the corrections to the energy spectrum obtained with the rectangular potential and to take the diffuse tailing of the interface into the account.

The introduction of the smooth functions $m(r)$ and $U(r)$ for $\mathrm{HgS} / \mathrm{CdS}$ quantum dots with $R>15 a_{\mathrm{HgS}}$ and $\mathrm{GaAs} / \mathrm{Al}_{0.4} \mathrm{Ga}_{0.6}$ QDs with the radii $R>20 a_{\mathrm{GaAs}}$ causes a shift of all energy levels into the region of higher energies.

It is established that the corrections to three lowest spherically-symmetric energy levels become closer to one another with increase in the size of QDs and weakly depend on the radii of big quantum dots.

The increase of the tailing parameter causes the increase of the corrections to the energy spectrum of an electron in a quantum dot.

\section{References}

1. M. Ciurla, J. Adamowski., B. Szafran, S. Bednarek, Modelling of confinement potentials in quantum dots // Physica E: Low-Dimens. Syst. Nanostr. 15, p. 162-168 (2002).

2. T. Nakamura, Modification of the image potential for the interfacial zone with varying dielectric constant // J. Phys. Soc. Jpn 52, p. 973-980 (1983).

3. V.I. Boichuk, R.Yu. Kubay, The potential energy of a charge near the surface of the spherical semiconductor microcrystal at the presence of intermediate layer of varying dielectric constant // Zhurn. Fiz. Doslidzhen' 3, p. $492-497$ (1999) (in Ukrainian).

4. V.I. Boichuk, R.Yu. Kubay, I.V. Bilynsky, The influence of the image potential on the electron energy spectrum in complicated spherical microcrystal $\mathrm{CdS} / \mathrm{HgS} / \mathrm{H}_{2} \mathrm{O}$ // Zhurn. Fiz. Doslidzhen' 3, p. 187-191 (1999) (in Ukrainian).

5. V.I. Boichuk, I.V. Bilynsky, R.Yu. Kubay, Energy spectrum of a charged near-surface complicated 
spherical heterostructure with taking into account noninertial polarization // Ukr. J. Phys. 45, p. 236$241(2000)$.

6. M. Tkach, V. Holovatsky, O. Voitsekhivska, M. Mikhalyova, Spectrum and interactions of quasiparticles in complicated spherical nanoheterosystems $\mathrm{CdS} / \mathrm{HgS} / \mathrm{ZnO} / \mathrm{H}_{2} \mathrm{O}$ // Electrochemical Society Proc. 25, p. 316-321 (1998).

7. N.V. Tkach, O.N. Voitsehivskaya, V.A. Golovatskii, M.Ya. Mikhal'ova, Spectrum of electron and holes in a complicated spherical semiconductor nanoheterosystem // Izv. Vuzov. Fiz., No 12, p. 5865 (1998).

8. M. Tkach, V. Holovatsky, O. Voitsekhivska, Ya. Berezovsky, Electron resonance states in opened spherical two-barrier nanoheterostructures // Ukr. J. Phys. 46, p. 859-864 (2001).

9. M. Tkach, V. Holovatsky, Lifetime of electron and hole quasistationary states in a spherical nanoheterosystem $\beta-\mathrm{HgS} / \beta-\mathrm{CdS} / \beta-\mathrm{HgS} / / U k r . J$. Phys. 45, p. $972-975$ (2000). 